\title{
Accumulated brisk walking and cardiovascular risk in an 'at risk' population: a preliminary investigation into the novel effects on HDL functionality
}

\author{
TM. Kearney ${ }^{1}$, J. McEneny², GW. Davison ${ }^{3}$, MJ. O’Kane ${ }^{4}$, MH. Murphy ${ }^{3}$ and AM. Gallagher ${ }^{1}$ \\ ${ }^{1}$ Northern Ireland Centre for Food and Health, University of Ulster, Coleraine BT52 1SA. ${ }^{2}$ Centre for Public \\ Health, Queen's University Belfast BT12 6BJ. ${ }^{3}$ Sport and Exercise Science Research Institute, University of \\ Ulster, Newtownabbey BT37 OQB. and ${ }^{4}$ Department of Clinical Chemistry, Altnagelvin Area Hospital, Londonderry \\ BT47 6SB, UK
}

Overweight/obesity now dominate the standard risk factors for cardiovascular disease (CVD) ${ }^{(1)}$. Regular physical activity, even without weight loss, is associated with reduced CVD-risk. It is recommended that adults undertake at least 150 min of moderate intensity activity per week, which can be accumulated in 10 min bouts. Emerging evidence suggests that high density lipoproteins (HDL) can become dysfunctional in the presence of inflammation, reducing its ability to protect against CVD. The inflammatory molecule serum amyloid A (SAA) displaces apolipoprotein AI from the HDL molecule, which may reduce LCAT activity, thus influencing reverse cholesterol transport. The effects of accumulated moderate intensity exercise (e.g. brisk walking) on HDL functionality in the absence of weight loss has yet to be determined in a population 'at risk' of CVD. This preliminary study investigated whether accumulated brisk walking in overweight/obese subjects (i.e. a group at risk of CVD) without weight loss could influence functional aspects of HDL.

77 overweight/obese sedentary individuals [19M, $58 \mathrm{~F}$; age $45 \cdot 6( \pm 6 \cdot 55)$ years; BMI $\left.29 \cdot 2( \pm 4 \cdot 27) \mathrm{kg} / \mathrm{m}^{2}\right]$ were randomly allocated to one of three groups; two groups completed $30 \mathrm{~min}$ of accumulated walking for 6 months with either weekly or monthly telephone support; the third group (control) performed stretching exercises. The walking groups were combined and telephone support included as a covariate. As previously reported, following 6 months of brisk walking, arterial stiffness was reduced in the walking groups along with an increase in fitness, effects which were sustained 4 months post-intervention ${ }^{(2)}$. These changes were observed despite no change in BMI, body fatness, blood pressure or fasting blood lipids (total cholesterol, HDL cholesterol, LDL cholesterol and triglycerides) across the study period ${ }^{(2)}$.To investigate the potential effects of accumulated brisk walking on functional aspects of HDL, HDL and $\mathrm{HDL}_{3}$ were isolated from fasting serum by rapid ultracentrifugation ${ }^{(3)}$. SAA (by ELISA), activities of HDL associated enzymes; LCAT and CETP (fluorometric assays) and PON-1 (spectrophotometric assay) were assessed in both fasting serum and isolated HDL sub-fractions. The lipid content of $\mathrm{HDL}_{2}$ and $\mathrm{HDL}_{3}$ was assessed spectrophotometrically. Only individuals with a complete dataset were included in analysis (Control group n19: Walking group n40). Data were analysed using repeated measures two-way analysis of variance (ANOVA) with $\mathrm{P}<0.05$ considered significant. No significant changes were observed for the lipid profile of $\mathrm{HDL}_{2}$ and $\mathrm{HDL}_{3}$ across the study period (all $\mathrm{P}>0.05$ ). Similarly, no significant change in the inflammatory marker SAA or in HDL-associated enzyme activities of CETP or PON-1were identified (all P >0.05). A trend towards increased serum LCAT activity

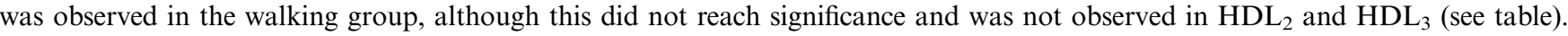

\begin{tabular}{|c|c|c|c|c|c|c|c|c|c|c|c|c|c|c|c|}
\hline \multirow{3}{*}{ LCAT Activity } & \multicolumn{4}{|c|}{ Serum $(390: 470 \mathrm{~nm})$} & \multirow[b]{3}{*}{$\mathrm{P}^{*}$} & \multicolumn{4}{|c|}{$\mathrm{HDL}_{2}(\mu \mathrm{ratio} / \mathrm{mg}$ protein) } & \multirow[b]{3}{*}{$\mathrm{P}^{*}$} & \multicolumn{4}{|c|}{$\mathrm{HDL}_{3}$ ( $\mu$ ratio/mg protein) } & \multirow[b]{3}{*}{$\mathrm{P} *$} \\
\hline & \multicolumn{2}{|c|}{ Control } & \multicolumn{2}{|c|}{ Walking } & & \multicolumn{2}{|c|}{ Control } & \multicolumn{2}{|c|}{ Walking } & & \multicolumn{2}{|c|}{ Control } & \multicolumn{2}{|c|}{ Walking } & \\
\hline & Mean & $\mathrm{SD}$ & Mean & SD & & Mean & SD & Mean & SD & & Mean & $\mathrm{SD}$ & Mean & $\mathrm{SD}$ & \\
\hline Baseline & $0 \cdot 9$ & $0 \cdot 18$ & 0.9 & $0 \cdot 38^{\mathrm{a}}$ & 0.98 & $32 \cdot 0$ & $12 \cdot 39$ & $28 \cdot 9$ & $14 \cdot 58$ & $0 \cdot 25$ & $0 \cdot 2$ & $12 \cdot 39$ & $0 \cdot 2$ & $0 \cdot 08$ & 0.52 \\
\hline End & $1 \cdot 0$ & $0 \cdot 22$ & $1 \cdot 0$ & $0 \cdot 42^{\mathrm{a}}$ & $0 \cdot 80$ & $31 \cdot 5$ & $8 \cdot 34$ & $29 \cdot 6$ & $13 \cdot 59$ & $0 \cdot 25$ & $0 \cdot 3$ & $8 \cdot 34$ & $0 \cdot 3$ & $0 \cdot 09$ & 0.75 \\
\hline Post & $1 \cdot 0$ & $0 \cdot 15$ & $1 \cdot 1$ & $0.52^{\mathrm{b}}$ & $0 \cdot 05$ & $32 \cdot 7$ & $11 \cdot 33$ & $29 \cdot 5$ & $14 \cdot 21$ & $0 \cdot 19$ & $0 \cdot 3$ & $11 \cdot 33$ & $0 \cdot 3$ & $0 \cdot 11$ & $0 \cdot 2$ \\
\hline$P^{+}$ & \multicolumn{2}{|c|}{0.69} & \multicolumn{2}{|c|}{0.06} & & \multicolumn{2}{|c|}{0.93} & \multicolumn{2}{|c|}{0.91} & & \multicolumn{2}{|c|}{$0 \cdot 16$} & \multicolumn{2}{|c|}{$0 \cdot 19$} & \\
\hline
\end{tabular}

Values are mean (SD) at baseline, end of intervention (end) and 4 months post-intervention (post)for control group (n19) and walking group (n40). ${ }^{*} \mathrm{P}\left(\right.$ time), ${ }^{+} \mathrm{P}($ within groups). Different superscripts indicate $\mathrm{P}<0.05$ between time points.

Retrospective power calculations based on this preliminary data indicate that 100 participants would be required to elicit a significant change to these measures of functional aspects of HDL, and future appropriately powered studies are needed to determine whether accumulated exercise in the absence of weight loss impacts on the antiatherogenic properties of HDL.

This work was supported by a grant from Northern Ireland Chest Heart and Stroke.

1. Nanchanhal K et al. (2005) Int J Obes. 29:317-23.

2. Kearney TM et al. (2014) J Am Soc Hypertension. 8:117-126.

3. McPherson et al. (2007) J Lipid Res. 48:86-95. 